\title{
ONIOM(QM:QM') Electrostatic Embedding Schemes for Photochemistry in Molecular Crystals
}

\author{
Miguel Rivera, Michael Dommett, and Rachel Crespo-Otero* \\ School of Biological and Chemical Sciences, \\ Queen Mary University of London, Mile End Road, London E1 4NS, UK \\ E-mail: r.crespo-otero@qmul.ac.uk
}

\begin{abstract}
Understanding photoinduced processes in molecular crystals is central to the design of highly emissive materials such as organic lasers and organic light-emitting diodes. The modelling of such processes is, however, hindered by the lack of excited state methodologies tailored for these systems. Embedding approaches based on the Ewald sum can be used in conjunction with excited state electronic structure methods to model the localised excitations which characterise these materials. In this article, we describe the implementation of a two-level ONIOM(QM:QM') point charge embedding approach based on the Ewald method, the ONIOM Ewald Embedded Cluster (OEEC) model. An alternative self-consistent method is also considered to simulate the response of the environment to the excitation. Two molecular crystals with opposing photochemical behaviour were used to benchmark the results with single reference and multireference methods. We observed that the inclusion of an explicit ground state cluster surrounding the QM region was imperative for the exploration of the excited state potential energy surfaces. Using OEEC, accurate absorption and emission energies as
\end{abstract}


well as $\mathrm{S}_{1}-\mathrm{S}_{0}$ conical intersections were obtained for both crystals. We discuss the implications of the use of these embedding schemes considering the degree of localisation of the excitation. The methods discussed herein are implemented in an open source platform (fromage, https://github.com/Crespo-Otero-group/fromage) which acts as an interface between popular electronic structure codes (Gaussian, Turbomole and Molcas). 


\section{Introduction}

Highly emissive organic crystals have great potential for the development of optoelectronic and photonic devices such as organic-light-emitting diodes and organic lasers.1 13 The electronic structure of the constituent monomers, intermolecular interactions and the electrostatic field in the crystal environment all contribute to the competition between radiative and nonradiative pathways, such as internal conversion and intersystem crossing. The exploration of excited state potential energy surfaces (PESs) in the solid state can help decipher the role of these interconnected factors and rationalise observed quantum yields.

Excitations in molecular crystals are normally localised over a few molecular units and can be strongly influenced by the periodic crystal environment. $\stackrel{4}{\text { This }}$ poses a challenge for traditional electronic structure methods, which have been designed to describe either highly localised or periodic delocalised electronic states. In this context, embedding techniques represent a viable option by combining higher quantum mechanical levels of theory to describe the excited region (QM) and more approximate methods for the crystal environment (QM' or MM).

Within the ONIOM scheme, the QM' method can be chosen to be plane-wave DFT ${ }^{6 / 7}$ for a natural description of the lattice periodicity, although this usually means sacrificing the electrostatic embedding. Correlated wavefunction-in-DFT periodic embedding approaches are a promising alternative. ${ }^{810}$ One of the most common approaches is to use cluster models to describe the periodic crystal. 11 13 The cluster is extracted from the atomic lattice positions and provides an energetic description of the short-range interactions with the QM region.

In the case of ionic or highly polar crystals, long-range interactions can be of great importance since the electrostatic potential is slowly and conditionally convergent.14 The long-range Coulomb interactions with distant atomic centres are therefore traditionally eval-

uated using Ewald summation techniques. $\frac{15}{17}$ The expression for the Ewald potential at position $r$ is: 


$$
V^{\text {Ewald }}(\boldsymbol{r})=\sum_{\boldsymbol{L} s} q_{s} \frac{\operatorname{erfc}\left(\gamma\left|\boldsymbol{r}-\boldsymbol{L}-\boldsymbol{R}_{s}\right|\right)}{\left|\boldsymbol{r}-\boldsymbol{L}-\boldsymbol{R}_{s}\right|}+\frac{4 \pi}{v_{c}} \sum_{\mathbf{G} \neq \mathbf{0}} \frac{1}{G^{2}} e^{-G^{2} / 4 \gamma^{2}}\left[\sum_{s} q_{s} e^{i \boldsymbol{G}\left(\boldsymbol{r}-\boldsymbol{R}_{s}\right)}\right]
$$

Where $\boldsymbol{L}$ and $\boldsymbol{G}$ are the real and reciprocal space lattice translations, $q_{s}$ are the charges of each site $s$ of the unit cell at positions $\boldsymbol{R}_{s}, \gamma$ is the Ewald constant and $v_{c}$ is the volume of the unit cell. Here, the direct sum electrostatic potential has been recast as a sum of two rapidly converging series. Short-range Coulomb terms are calculated in direct space and long-range interactions using a Fourier series in reciprocal space. To evaluate the Ewald potential on a lattice site $\boldsymbol{r}_{i}$, the self-potential of the charge must be subtracted to avoid a singularity which amounts to replacing the $\boldsymbol{L}=0$ and $s=i$ case of the first term of eq 1 with $-\frac{2 \gamma q_{i}}{\sqrt{\pi}} \cdot \frac{14118]}{20}$

When considering embedded finite cluster models, the electrostatic embedding can be modified to reflect the Ewald potential. In this case, the electrostatic interactions affecting the QM region extend beyond just the short-range and up to the infinitely large in a periodic system. Klintenberg et al. developed a methodology where a large array of point charges is fitted to reproduce the exact Ewald potential inside the QM region of a cluster model $\frac{15121 / 22}{[1}$ This procedure has been used for the investigation of ionic crystals and the calculation of NMR parameters in organic crystals. $\frac{1512324}{1}$ Sokol et al. have implemented a related method in Chemshell to model defects in ionic materials. ${ }^{2526}$ An alternative is the procedure proposed by Abrenkov and Sushko, where compensating point charges are added within unit cells to approach the Ewald potential. 2728

Ewald embedding methods have been used with QM:MM and ONIOM approaches allowing the evaluation of the short-range non-Coulombic interactions. ${ }^{[29}[4]$ However a simpler variant is the Point Charge Embedding approach (PCE) where only the Coulomb interactions are considered, using point charges, and non-electrostatic interactions are neglected.5115 The performance of these methods for the investigation of excited states PESs of molecular 
crystals is relatively unexplored. Recently, Ciofini and co-workers ${ }^{35} \sqrt[37]{3}$ have implemented an Ewald PCE scheme based on the method proposed by Derenzo et al.. ${ }^{22}$ In order to consider mutual polarisation effects of the crystal environment, a self-consistent algorithm was employed in the investigation of a crystal displaying aggregation-induced emission. $\stackrel{36}{\text { Self- }}$ consistent schemes are typical tools used in QM:MM schemes when the polarisation of the environment is important. 38,40

Herein we present the implementation of Ewald embedding approaches for the description of PESs of molecular crystals, with specific focus on the treatment of excited state minima and conical intersections. We show that due to the lack of short-range non-Coulombic interactions, geometry optimisation with the PCE method can be extremely problematic. As a solution, we implement an Ewald-embedded QM:QM' cluster model that can be used to explore the PES of flexible molecules. We assess the efficacy of these schemes with two crystals based on 2'-hydroxychalcone (HC1 and HC2, shown in Figure 1). These molecules undergo excited state intramolecular proton transfer (ESIPT), where the large changes in electronic structure in the excited state pose a challenge to embedding methods.

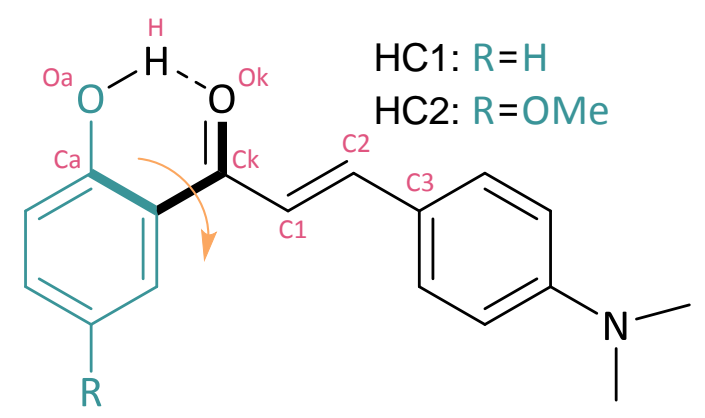

Figure 1: Molecular diagram of the 2'-hydroxychalcone derivatives, HC1 and HC2. The access to conical intersections for theses molecules is centered around the rotation of the blue group about the dihedral angle shown in bold. The notable atoms with large partial charge are labelled in pink.

We have previously investigated $\mathbf{H C 1}$ and $\mathbf{H C 2}$ in the context of aggregation induced emission (AIE). $\stackrel{4142}{ }$ The population of the keto $\left(\mathbf{K}^{*}\right)$ and enol $\left(\mathbf{E}^{*}\right)$ excited states depends on the identity of the substituents and crystal packing. ${ }^{42}$ HC1 displays emission in the 
crystal with promising properties to be used in solid state lasers $\frac{43}{43}$ and predominantly forms herringbone-type aggregates. In contrast, HC2's decay is mainly non-radiative and its crystal structure features mainly $\pi$-stack dimers. Their PESs were found to be particularly sensitive to the electrostatic environment. The AIE character of $\mathbf{H C 1}$ can be understood using the Restricted Access to Conical Intersections (RACI) model ${ }^{44[45}$ wherein upon aggregation the energy of the $\mathrm{S}_{1}-\mathrm{S}_{0}$ conical intersections increases, thereby blocking nonradiative deactivation pathways and enhancing the emissive response.

The paper is organised as followed. First, we present the different embedding models and the details of their implementation. Next, we define how to choose the size of the high-level QM region, an important step in the division of the cluster regions. We then determine the effect of different point charge embedding schemes and assess their overall performance. In our conclusions, we suggest a protocol for researchers studying excited states in molecular crystals. The presented methodologies are implemented in a new open-source platform: fromage (FRamewOrk for Molecular AGgregate Excitations).

\section{Embedding Schemes}

We consider two electrostatic Ewald embedding approaches to investigate excited states in molecular crystals: PCE and a two-level ONIOM(QM:QM') model. For the PCE approach, where only the Coulombic interactions are considered, we adopt a strategy similar to that

proposed by Wilbraham et al. ${ }^{[35}$ The atomic charges were obtained using the Ewald program from Derenzo et al. ${ }^{22}$ after being modified to allow non-integer charge values. The effect of the polarisation of the environment was considered for both methods within a self-consistent embedding algorithm. These approaches were implemented in fromage, the source code and the documentation are available online. $\underline{46 / 47}$

In the Ewald program, 22 an array of about $10^{4}$ charges is generated from a supercell. Three zones are defined, the central region (zone $\mathbf{I}$ ) is where the highest level of theory will 
be used. It is spherically surrounded by a buffer region (zone II) of approximately 500 point charges. Charges of both zone I and II are held constant. The rest of the charges (zone III) are altered to reproduce the Ewald potential in the central and buffer regions. The algorithm removes any artificial dipole moment introduced in the procedure. A detailed description of the method and the corresponding program can be found in Reference 22.

The implementation of PCE in fromage consists of electronic structure calculations at zone I atomic sites, embedded in the atomic charges of zones II and III. For clarity, we refer to zone II and III charges as Ewald charges. Excited state energies are obtained

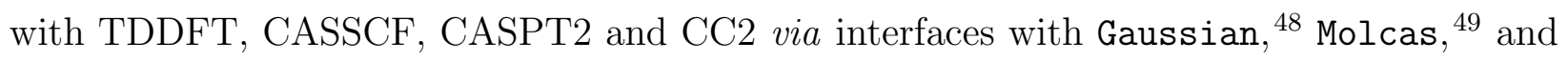
Turbomole. ${ }^{50}$ An interface with $\mathrm{DFTB}^{+51}$ is under development. The atomic charges can be obtained from molecular or periodic crystal calculations. We consider RESP, Mulliken and NBO from molecular calculations and RESP, Mulliken, Hirshfeld and AIM charges from periodic calculations. Currently, atomic charges can be read from Gaussian and CP2K.

fromage provides tools for the exploration of PESs of molecular crystals. The L-BFGS minimisation algorithm is used to locate stationary points. A complete characterisation of excited state potential energy surfaces in molecular crystals require the description of conical intersections. We have implemented the penalty function method of Levine et al. 52 to optimise Minimal Energy Conical Intersection (MECI) geometries. In contrast with other methods, $\frac{53}{5}$ this approach does not require nonadiabatic coupling vectors. A function of the averaged $\mathrm{S}_{1}$ and $\mathrm{S}_{0}$ energies $\left(\bar{E}_{1-0}\right)$ and the $\mathrm{S}_{1}-\mathrm{S}_{0}$ energy gap $(\Delta E)$ is minimised:

$$
F=\bar{E}_{1-0}+\sigma \frac{\Delta E^{2}}{|\Delta E|+\alpha}
$$

where $\sigma$ is a Lagrangian multiplier and $\alpha$ is a parameter such that $\alpha \ll|\Delta E|$. This algorithm is implemented in fromage for CASSCF, CC2 and TDDFT electronic methods. We would like to emphasise that even when multireference quantum methods are preferable for modelling $\mathrm{S}_{1}-\mathrm{S}_{0}$ crossings,,$\underline{5455}$ in many cases single-reference methods can provide a qualitative 
description of these regions of the PES. Crossings involving excited states can be described with single-reference methods such as TDDFT. 55 Nonadiabatic dynamics simulations with these methods have shown for multiple systems that methods such as $\mathrm{ADC}(2)$ and $\mathrm{CC} 2$ can provide reasonable results. $\frac{56157}{}$ In the case of TDDFT, a careful selection of the functional is required. $[58159$ Considering the computational cost of multireference methods and the sensitivity of their active space, it can at times be necessary to resort to single-reference methods. However, their performance near $\mathrm{S}_{1}-\mathrm{S}_{0}$ crossings should be carefully tested by comparison with multireference calculations.

Geometry optimisation and conical intersection search become problematic within the PCE scheme because of the lack of short-range non-Coulombic interactions which results in overpolarisation effects (see section 4.2). To overcome these limitations, we formulate an $\mathrm{ONIOM}^{60}$ Ewald Embedded Cluster (OEEC) model. It is devised as an extension of the commonly used ONIOM Embedded Cluster model (OEC) which usually only includes electrostatic embedding up to the range of the cluster. We consider a QM:QM' scheme rather than QM:MM to avoid the need for specific parameterisation.

A graphical representation of our EEC model is shown in Figure 2. The EEC model is comprised of two regions, the central region $\mathbf{1}$ (corresponding to zone $\mathbf{I}$ in the Ewald program) and nearest-neighbour molecules (2). Region $\mathbf{2}$ should be large enough to include the most important short-range non-electrostatic interactions with the QM cluster. The buffer region defined for Ewald (zone II) does not necessarily correspond to region 2.

Identically to those with PCE, excited state calculations of region $\mathbf{1}$ are embedded in Ewald charges and are performed at the highest level of theory — with a QM method — yielding the energy $E_{Q M}^{E w}(\mathbf{1})$. Two ground state energy calculations are further performed at a QM' level of theory: the energies of the cluster composed of regions $\mathbf{1}$ and $\mathbf{2}\left(E_{Q M^{\prime}}(\mathbf{1} \cup \mathbf{2})\right)$ and the energy of region $\mathbf{1}$ electrostatically embedded in the charges of $\mathbf{2}\left(E_{Q M^{\prime}}^{E E}(\mathbf{1})\right)$. The total energy in the cluster is calculated as follows: 


$$
E_{Q M: Q M^{\prime}}^{E w}(\mathbf{1} \cup \mathbf{2})=E_{Q M}^{E w}(\mathbf{1})+E_{Q M^{\prime}}(\mathbf{1} \cup \mathbf{2})-E_{Q M^{\prime}}^{E E}(\mathbf{1})
$$

The hybrid gradients are defined accordingly.
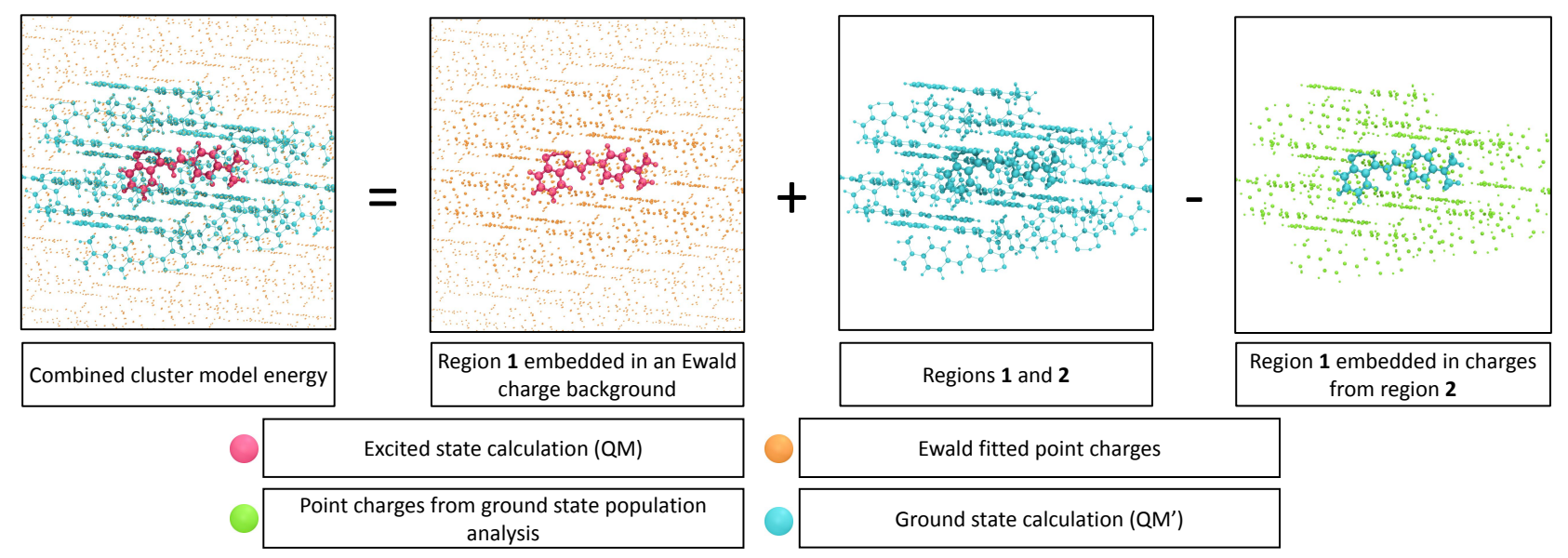

Figure 2: Visual representation of the main energy equation for the Ewald Embedded Cluster model.

In the OEEC scheme, Coulombic interactions of any distance between region $\mathbf{1}$ and the crystal are described at the higher level of theory (QM). For excited state calculations, this represents the interaction between an excited central region and the environment in the ground state, unless particular charges are considered in the Ewald algorithm (vide infra). In contrast, the short-range non-Coulombic interactions between $\mathbf{1}$ and $\mathbf{2}$ are considered at the QM' ground state level, which recovers some of the short-range contributions and improves the description provided by PCE. Since non-Coulombic interactions are considered in the ground state, for fixed geometries the energy gaps are equivalent to those obtained with the PCE. The selection of the QM' level of theory depends on the available computational resources. Previous studies on truncated cluster models have shown that low levels of theory such as HF/STO-3G achieve accurate results. [6]12[35|36|61

The choice of charges in the embedding of $E_{Q M^{\prime}}^{E E}(\mathbf{1})$ in eq 3 should provide an approximate cancellation of the Coulombic interactions between regions $\mathbf{1}$ and $\mathbf{2}$ in the ground state (see Supporting Information). ${ }^{62}$ We therefore use charges obtained from a QM' population 
analysis in the embedding of $E_{Q M^{\prime}}^{E E}(\mathbf{1})$, whilst using charges from a QM ground state calculation in the embedding of $E_{Q M}^{E w}(\mathbf{1})$ for an accurate representation of the lattice electrostatic potential. In contrast, traditional ONIOM schemes use the same partial charge values for both embeddings. This is done in order to mitigate overpolarisation effects stemming from the use of point charges; these effects being particularly severe when the inter-region boundary crosses a bond and link atoms need to be used. However in the cases discussed herein, the region boundary is defined inter- rather than intramolecularly and most intermolecular contacts are larger than $4 \AA$, which allows us to select charges of the highest quality in the embedding of $E_{Q M}^{E w}(\mathbf{1})$. This situation can be generalised to any organic molecular crystal with similar or higher sparsity in packing. Further extensions of these methods can be implemented to reduce artificial polarisation ${ }^{63}$ and make the methods useful for more dense systems. We are already working on this direction.

The alternative scheme where Ewald charges are used for the QM' calculations should provide a worse compensation of the inter-region Coulombic interactions. For testing purposes, we have also implemented this scheme in fromage $\left(E_{Q M^{\prime}}^{E w}(\mathbf{1})\right.$ as the final term of eq 3$)$. Nevertheless, the results obtained with this embedding scheme are similar to those obtained with the cluster charges for HC1, possibly due to a cancellation of errors. The absorption energies are only deviated by $0.01 \mathrm{eV}$ from those obtained with the original scheme. In the case of the emission energies from the $\mathbf{K}$ form, the value obtained with this version of OEEC is $2.24 \mathrm{eV}$ which is in relative good agreement with the results obtained with other schemes (Table 3).

In order to consider the response of the environment to the excitation and recover mutual polarisation effects, we employ the extension of self-consistent Ewald embedding to excited states proposed by Wilbraham et al. $\frac{35}{3}$ Mutually polarising embedding methods have been applied to a number of ground state systems. ${ }^{15 / 24|64| 65 \mid}$ In the self-consistent approach, a QMlevel calculation is carried out on a quantum cluster. A population analysis is then applied and the charge values are re-assigned to the equivalent positions in the crystal. Those charges 
are then fitted using Ewald and another QM calculation is carried out. The loop between Ewald fitting and population analysis is repeated until convergence of the atomic charges. The new charge background is used for the electrostatic embedding of $\mathbf{1}$. In fromage, the self-consistent approach is implemented for the PCE and the QM/QM' approaches (SC-PCE and SC-OEEC). We consider two versions which may represent different physical situations in the crystal (discussed in section 4.1). The first, $\mathrm{SC}-\mathrm{PCE}-\mathrm{S}_{1}$, closely corresponds to the embedding proposed by Wilbraham et al.; it uses excited state charges as an initial charge background and iterates with excited state population analyses. The second, SC-PCE-S has a ground state initial charge background and performs ground state population analyses.

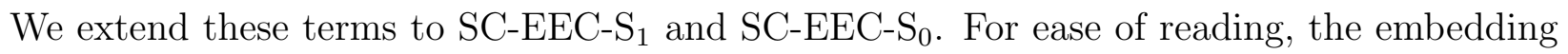
models are listed in Table 1 .

For SC-PCE-S ${ }_{1}$, the convergence can be sped up by starting the loop from a ground state population analysis embedded in ground state Ewald charges. The final background was found to be very similar, with an RMSD of $10^{-5} \mathrm{e}^{-}$for atomic charges. Another alternative is to perform the loop on a molecule which has already been optimised in the excited state using OEEC. In this case, the equilibration of the charge background is made to match the excited state minimum, however this implies assigning charges from an excited state minimum configuration to region $\mathbf{2}$ molecules which are in their ground state minimum geometry. 
Table 1: Embedding models used in this study

\begin{tabular}{|c|c|c|}
\hline Acronym & Full name & Description \\
\hline PCE & Point Charge Embedding & $\begin{array}{l}\text { Point charge embedding fitted to } \\
\text { match the Ewald potential }\end{array}$ \\
\hline SC-PCE-S ${ }_{1}$ & $\begin{array}{l}\text { Self-Consistent Point Charge Em- } \\
\text { bedding }\end{array}$ & $\begin{array}{l}\text { PCE computed self consistently } \\
\text { in } \mathrm{S}_{1}\end{array}$ \\
\hline SC-PCE-S ${ }_{0}$ & $\begin{array}{l}\text { Self-Consistent Point Charge Em- } \\
\text { bedding }\end{array}$ & $\begin{array}{l}\text { PCE computed self consistently } \\
\text { in } S_{0}\end{array}$ \\
\hline OEC & ONIOM Embedded Cluster & $\begin{array}{l}\text { QM:QM' ONIOM cluster model } \\
\text { with the QM region embedded in } \\
\text { charges from the QM' region }\end{array}$ \\
\hline OEEC & $\begin{array}{l}\text { ONIOM Ewald Embedded Clus- } \\
\text { ter }\end{array}$ & $\begin{array}{l}\text { OEC with the QM region embed- } \\
\text { ded in charges from PCE }\end{array}$ \\
\hline SC-OEEC-S $_{1}$ & $\begin{array}{l}\text { Self-Consistent ONIOM Ewald } \\
\text { Embedded Cluster } \mathrm{S}_{1}\end{array}$ & $\begin{array}{l}\text { OEC with the QM region embed- } \\
\text { ded in charges from SC-PCE- } \mathrm{S}_{1}\end{array}$ \\
\hline SC-OEECS $_{0}$ & $\begin{array}{l}\text { Self-Consistent ONIOM Ewald } \\
\text { Embedded Cluster } \mathrm{S}_{0}\end{array}$ & $\begin{array}{l}\text { OEC with the QM region embed- } \\
\text { ded in charges from SC-PCE- } \mathrm{S}_{0}\end{array}$ \\
\hline
\end{tabular}

Figure 3 describes the structure of fromage. The charge background can be chosen to be computed self-consistently and the geometry optimisation can be set to search for ground and excited state minima or MECI. Currently, region 2 is fixed in place during geometry optimisation, although full cluster relaxation ${ }^{53}$ is under development. For SC-OEEC, to recover point charges of the highest quality, the molecule of interest in the unit cell is first relaxed with OEEC. Furthermore the self-consistent charge background is computed only for the first step, at the ground state OEEC geometry, in order to maintain a consistent PES throughout the relaxation.

\section{Computational Details}

The crystal structures of $\mathbf{H C 1}$ and $\mathbf{H C 2}$ were optimised using PBE-D2 as implemented in Quantum Espresso. ${ }^{66}$ The plane wave cutoff was 30 Ry and the k-point meshes were $2 \times 3 \times 2$ 


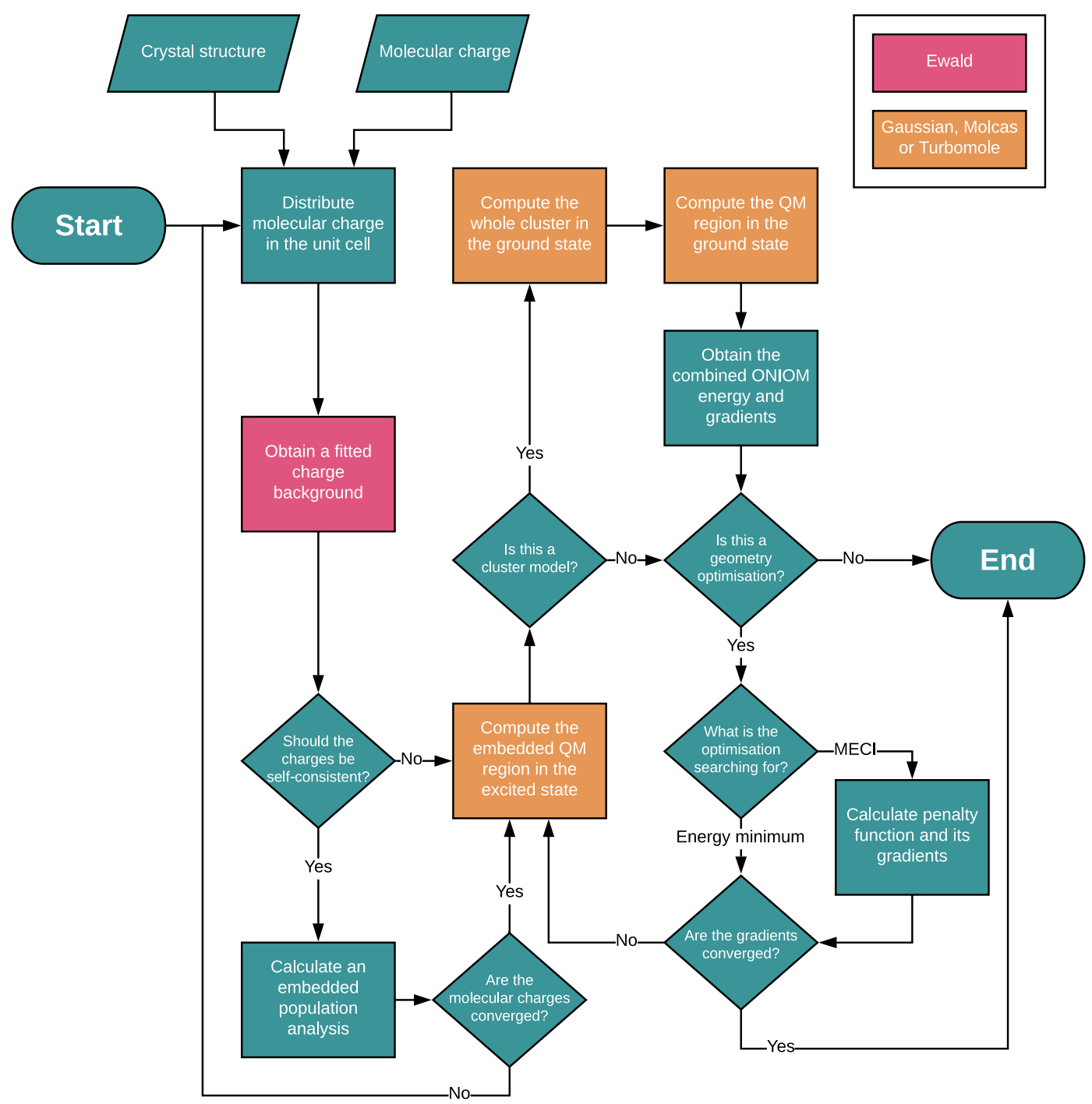

Figure 3: Flowchart of a calculation using the ONIOM Ewald Embedded Cluster (OEEC) and Self-Consistent ONIOM Ewald Embedded Cluster (SC-OEEC) models. The electronic program can be chosen by the user. 
and 2x2x1 respectively, in accordance with the shapes of the unit cells. Subsequently, a single point PBE-D2/DZVP calculation was carried out using CP2K ${ }^{67}$ to extract RESP, Hirshfeld and Mulliken periodic charges. For AIM charges, an external program developed by Henkelman et al. was used to process the Quantum Espresso DFT charge density. $\underline{68} 7 \underline{71}$ Molecular RESP charges were first calculated at HF/3-21G(p) level for comparison with our previous ONIOM (QM:AMBER) calculations. ${ }^{41}$ Every other molecular population analysis (NBO, Mulliken, RESP for OEEC and SC-OEEC models) used $\omega$ B97X-D/6-311++G(d,p) as implemented in Gaussian.

For the seven charge schemes, 1000 checkpoints were sampled in the quantum cluster and 500 points had their value fixed to create a buffer region. The total charge background was comprised of 64 unit cells for HC1 and 32 for HC2. These numbers were chosen so as to create a sufficient amount of point charges $^{[22}$ at least 10000 - while keeping an isotropic distribution in accordance with the shape and size of each unit cell.

Both molecular crystals were then investigated using a hierarchy of models. First, PCE was used with all of the charge types described above on a single QM-level monomer. When possible, the excited state geometries were optimised with TD- $\omega$ B97X-D/6-311++G(d,p). Next, the cluster models were introduced, using RESP charges from $\omega$ B97X-D/6-311++G(d,p) in the embedding of $E_{E w}^{Q M}(\mathbf{1})$. OEC, OEEC and SC-OEEC were all employed on a single monomer of the crystal embedded in a cluster of 21 molecules for HC1 and 16 molecules for HC2. The excited state minima and $\mathrm{S}_{1}-\mathrm{S}_{0} \mathrm{MECI}$ were found using fromage. For the location of $\mathrm{S}_{1}-\mathrm{S}_{0}$ MECI, the parameters in eq 2 were initially set to 0.02 Hartree for $\alpha$ and 3.5 for $\sigma . \sigma$ was then increased if the gap was found to be insufficiently small after optimisation of $F$.

For the comparison of different points along the potential energy surface, we use a fixed charge background. This avoids varying classical energy contributions due to charge-charge interactions and different Ewald constants. $\frac{14}{1 n}$ this article, we used the charge background obtained for the FC conformation, although for crystals with significant Frenkel exciton 
occurrences, $\mathrm{S}_{1}$ self-consistent charges could provide a better description of the excited states. All backgrounds are available in fromage, leaving the choice up to the user.

Overall, the QM methods employed were TD- $\omega$ B97X-D/6-311++G(d,p) using Gaussian, RI-CC2/TZVP and RI-CC2/SV(P) using Turbomole and SA-2-CASSCF(12,11)/6-31G(d) and MS-2-CASPT2(12,11)/6-31G(d) using Molcas; all with PCE, OEEC and SC-OEEC. The QM' method was HF/STO-3G using Gaussian and the low level embedding charges

of $E_{Q M^{\prime}}^{E E}(\mathbf{1})$ were accordingly chosen to be from RESP calculations at the same level of theory. For the self-consistent population analysis procedure, a convergence criterion of $0.001 e^{-}$for the mean deviation of charge values between subsequent steps was chosen. Where necessary, under-relaxation was employed with a damping factor of 0.75 to address convergence issues in the self-consistent loop such as divergence or oscillation. For excited state self consistent backgrounds, using initial charges from an isolated excited state molecule or an Ewald embedded ground state molecule yielded the same final background although the latter method converged in fewer steps.

For comparison, single monomers were also optimised in the ground and excited states using TD- $\omega$ B97X-D/6-311++G(d,p) in vacuum and using Polarisable Continuum Models (PCM) and Self-Consistent PCM (SC-PCM) with a dichloromethane (DCM) solvent as implemented in Gaussian. Exciton couplings were computed using the diabatisation scheme proposed by Aragó and Troisi, which considers short and long-range contributions. ${ }^{4}$

\section{Results}

\subsection{Localisation of the Excitation: Size of the QM Region}

The use of embedding techniques for excited states calculations in molecular crystals presumes the localisation of the excitation over a few molecular units. However, the degree of localisation is often unclear and unpredictable, conflicting with the intrinsic truncation of a cluster model. Therein lies the necessity for different kinds of embedding techniques which 
represent different physical situations. Before comparing the effect of these techniques, we wish to clarify how they relate to exciton localisation in our model systems.

In the case of OEEC, the Ewald charges arise from a ground state population analysis. Consequently, this approach represents a localised excitation in region $\mathbf{1}$ before the environment has responded to the change in electronic density. To instead represent the extreme situation where all molecules are excited simultaneously and are mutually responsive, charges

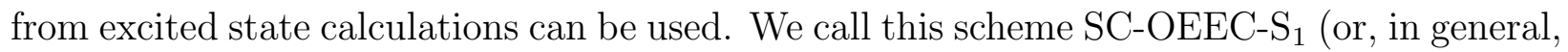
SC-OEEC-S ${ }_{n}$ ). If the molecules in the QM' region are considered to be in the ground state and the $\mathrm{S}_{0}$ charges are self-consistently updated and alternative $\mathrm{SC}-\mathrm{OEEC}-\mathrm{S}_{0}$ scheme can be defined. It is expected that the $\mathrm{SC}-\mathrm{OEEC}-\mathrm{S}_{1}$ scheme will perform better in systems where excitation is highly delocalised and the $\mathrm{S}_{1}$ electron density is significantly different from the ground state. We have implemented all these schemes in fromage so that the user can select the most suitable scheme for the system under investigation. The degree of localisation of the excitation in a molecular crystal will depend on the exciton coupling with neighbouring molecules and the experimental conditions for absorption.

In order to investigate the excitonic features of the excited state electron densities of the HC1 and HC2 crystals, we consider a tetramer (Figure 4) embedded in ground state Ewald charges as a reference. This model includes the short-range Coulomb interactions between the central and three surrounding molecules explicitly and thus should provide a benchmark to evaluate the ability of the different embedding schemes to describe the excited states considering a smaller QM region. Note that in contrast with the monomer, where the bright state is $\mathrm{S}_{1}$, for the tetramer the bright states are $\mathrm{S}_{4}$ and $\mathrm{S}_{5}$ for $\mathbf{H C 1}$ and $\mathbf{H C 2}$ respectively.

Figure 5 shows the $\mathrm{S}_{n}-\mathrm{S}_{0}$ density differences obtained for the bright state at FranckCondon geometry $(\mathbf{F C})$ and the $\mathbf{K}^{*} \mathrm{~S}_{1}$ excited state minimum geometries. The plots for the first five excited states can be found in the Supporting Information. When considering a full tetramer in the excited state, the bright states of $\mathbf{H C 1}$ and $\mathbf{H C 2}$ are $\mathrm{S}_{4}$ and $\mathrm{S}_{5}$ respectively, whereas for single monomers, they are both $\mathrm{S}_{1}$. An important degree of localisation is 

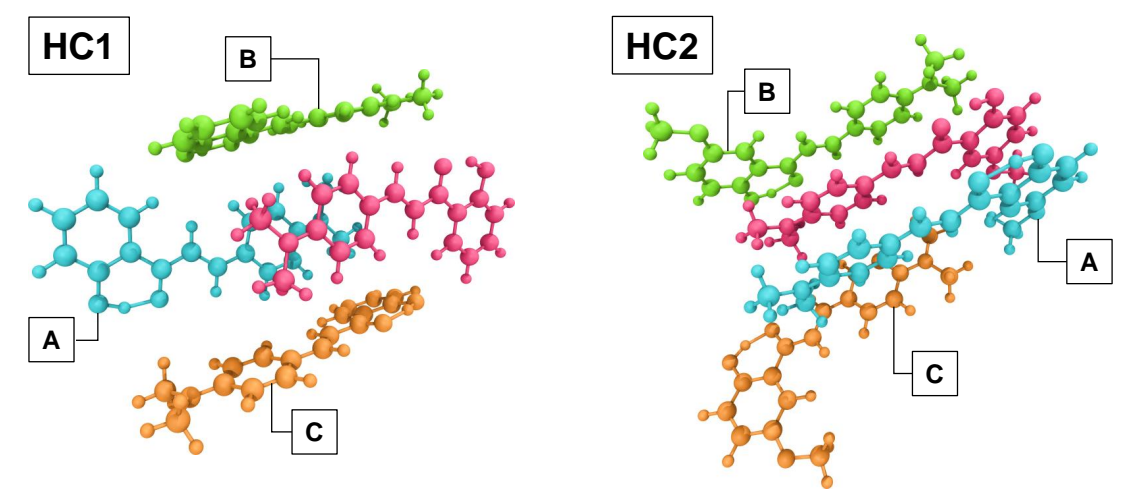

Figure 4: Selected tetramer configurations from both crystals. The molecule in pink is optimised using OEEC.

observed on monomers and dimers for both crystals, despite four molecules being included in the QM region. Consequently, we expect that with embedding charges of sufficient quality, a QM region of one or two molecules would obtain accurate excited state energies.

Excitations in $\mathbf{H C 1}$ are more localised than in $\mathbf{H C 2}$, which correlates with the larger exciton couplings obtained for the latter. In the case of $\mathbf{H C 1}$, only the coupling with molecule $\mathrm{B}$ is larger than $0.1 \mathrm{eV}$ (Supporting Information). For both crystals, in the $\mathbf{K}^{*}$ minimum, the excitation is clearly localised in the central molecule, which suggests that schemes such as OEEC and SC-OEEC-S S $_{0}$ could be best suited to describe this kind of situations (see discussion in the next sections). Additionally, the QM region with only one monomer should be able to describe emission from the $\mathbf{K}^{*}$ form, which is confirmed by the evolution of the energies with the size of the region (see section 4.3).

\subsection{Point Charge Embedding: Electrostatic Effects in the Crystal}

In this section, we analyse the performance of the PCE model and the effect of using different charges for the description of excited states in the HC1 crystal. Our analysis is based on the results obtained with a monomer in the QM region.

The experimental absorption in the solid state shows two bands which have previously been attributed to absorption from the $\mathbf{E}$ and $\mathbf{K}$ forms. $\stackrel{43172}{ }$ Our calculations show that 


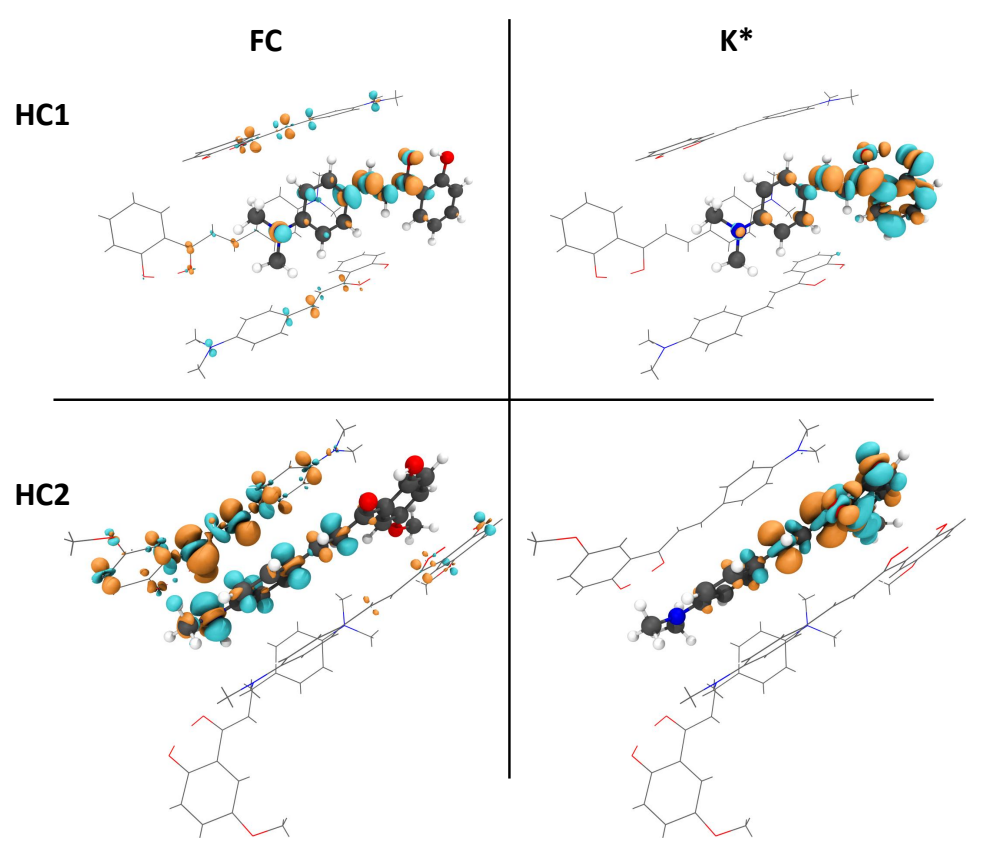

Figure 5: $\mathrm{S}_{n}-\mathrm{S}_{0}$ density differences obtained at TD- $\omega$ B97X-D/6-311++G(d,p) level of theory for the tetramer model (excited state density is shown in orange, ground state in blue). For the FC geometries, the tetramer's bright states were considered ( $n=4$ for HC1 and $n=5$ for HC2); for $\mathbf{K}^{*}, n=1$. All configurations were obtained by optimising the geometry of the central molecule with OEEC. 
Table 2: Absorption, emission and K-MECI energies (in eV) of $\mathbf{H C 1}$ embedded in different types of Ewald point charge arrays. Unless specified the geometries were obtained at the ONIOM(TD- $\omega$ B97X-D/6-311++G(d,p):AMBER) level of theory. K-MECI energies are relative to the ground state energy of the Franck-Condon (FC) minimum. ${ }^{42}{ }^{\dagger}$ Optimised geometries within the PCE environment. ${ }^{\dagger}{ }^{\dagger}$ Geometry optimised in vacuum

\begin{tabular}{|c|c|c|c|c|c|c|}
\hline \multirow[t]{2}{*}{ Method } & \multicolumn{2}{|c|}{ Charges } & \multirow{2}{*}{$\frac{\text { Absorption }}{\mathrm{FC}(\mathrm{E})}$} & \multicolumn{2}{|c|}{ Emission } & \multirow{2}{*}{$\frac{S_{1}-S_{0}}{\text { K-MECI }}$} \\
\hline & Type & Basis & & $\mathbf{E}^{*}$ & $\mathbf{K}^{*}$ & \\
\hline \multicolumn{7}{|c|}{ Molecular } \\
\hline \multirow{7}{*}{$\begin{array}{l}\text { TD- } \omega \text { B97X-D/ } \\
6-311++G(d, p)\end{array}$} & NBO & & 3.28 & 3.10 & 2.67 & 4.35 \\
\hline & RESP & & 3.30 & 3.12 & 2.66 & 4.41 \\
\hline & RESP (SC-PCE-S $\left.{ }_{1}\right)$ & $6-311++\mathrm{G}(\mathrm{d}, \mathrm{p})$ & 3.09 & 2.96 & 2.65 & 4.72 \\
\hline & $\mathrm{RESP}^{\dagger}$ & & 3.37 & - & 2.69 & 4.37 \\
\hline & Mulliken & & 1.56 & 1.51 & 1.47 & 4.42 \\
\hline & Mulliken & $3-21 \mathrm{G}(\mathrm{d})$ & 3.29 & 3.11 & 2.70 & 4.76 \\
\hline & Mulliken & $6-31 G(d)$ & 3.35 & 3.16 & 2.70 & 4.68 \\
\hline \multirow{3}{*}{$\begin{array}{l}\text { RI-CC2/SV(P) } \\
\text { RI-CC2/TZVP }\end{array}$} & RESP & \multirow{2}{*}{$6-311++\mathrm{G}(\mathrm{d}, \mathrm{p})$} & 3.11 & 2.95 & 2.35 & 3.82 \\
\hline & RESP & & 2.98 & 2.82 & 2.29 & 3.56 \\
\hline & \multicolumn{2}{|c|}{ Crystal } & & & & \\
\hline \multirow{5}{*}{$\begin{array}{l}\text { TD- } \omega \text { B97X-D/ } \\
6-311++G(d, p)\end{array}$} & RESP & & 3.33 & 3.15 & 2.68 & 4.32 \\
\hline & AIM & & 3.35 & 3.16 & 2.68 & 4.30 \\
\hline & Hirshfeld & DZVP & 3.43 & 3.23 & 2.68 & 4.56 \\
\hline & Hirshfeld $^{\dagger}$ & & 3.50 & 3.20 & 2.28 & 2.89 \\
\hline & Mulliken & & 2.95 & 2.85 & 2.64 & 4.20 \\
\hline \multirow{2}{*}{$\begin{array}{l}\text { TD- } \omega \text { B97X-D/ } \\
6-311++G(d, p) \\
\end{array}$} & No charges & - & 3.52 & 3.31 & 2.67 & 4.76 \\
\hline & Vacuum $^{\dagger \dagger}$ & - & 3.65 & 3.28 & 0.36 & 2.84 \\
\hline- & Experimental $^{[43 \mid 72]}$ & - & $2.9,3.3$ & - & $1.7-1.9$ & - \\
\hline
\end{tabular}


neither the crystal composed of $\mathbf{K}$ molecules nor the one with $\mathbf{K}$ surrounded by $\mathbf{E}$ molecules are stable in the solid state. Additionally, the experimental crystal structure does not seem to be consistent with a significant population of the $\mathbf{K}$ form in the ground state. ${ }^{[2]}$ Taking this into account, the presence of $\mathbf{K}$ in the ground state seems to be associated with dynamic processes activated in the experimental conditions. For example, at room temperature, large amplitude motions of the proton along the H-bonded bridge can reduce the $\mathrm{S}_{1}-\mathrm{S}_{0}$ energy gap to $2.76 \mathrm{eV}$, considering vibrational broadening. Additionally, given the ultrafast nature of the proton transfer in the solid state $\left(3 \mathrm{ps}^{(72}\right)$, fast absorption from $\mathbf{K}$ forms generated in the excited state could be also possible. The dynamic nature of these processes is in line with the broad structure of the low energy band. Our focus is the analysis of the higher energy band which corresponds to the absorption in the $\mathbf{E}$ form.

To estimate the effect of vibrational broadening on the position of the absorption maximum, we use the nuclear ensemble method ${ }^{[73}$ as implemented in Newton- $-X^{[74}$ with TD- $\omega$ B97X$\mathrm{D} / 6-311++\mathrm{G}(\mathrm{d}, \mathrm{p})$ embedded in RESP charges. The position of the $\mathbf{E}$ absorption maximum (3.21 eV, $0.1 \mathrm{eV}$ shift with respect to the vertical excitation with the same method) is in excellent agreement with the experimental value (Supporting Information).

To directly evaluate the effect of charges of different origin, we compare absorption, emission (from $\mathbf{E}^{*}$ and $\mathbf{K}^{*}$ forms) and $\mathrm{S}_{1}-\mathrm{S}_{0} \mathrm{MECI}$ energies with Ewald embedding. Given that the MECI associated with the enol pathway was consistently found to be at least 4 eV higher in energy than its keto counterpart, we will focus on the K-MECI deactivation pathway. The results are summarised in Table 2, In order to directly compare the impact of different charge partition schemes, we use the same geometry throughout, obtained at the $\operatorname{ONIOM}($ TD- $\omega$ B97X-D/6-311++G(d,p):AMBER) level of theory. $\cdot$. 22

Excited state calculations with PCE using non-Mulliken charges predict the maximum of absorption with close agreement to the experimental value of $3.3 \mathrm{eV}$. Overall, the effect of the embedding is to shift absorption to the red with respect to the energy obtained in vacuum $(3.65 \mathrm{eV})$. For calculations at fixed geometries, there is no significant dependence on 
whether the charges are obtained from molecular or crystal calculations. In particular, the energies obtained using RESP charges are consistent between the molecular and the crystal descriptions. In the context of the molecular organic crystals, this is not surprising as RESP charges are designed to match the electrostatic potential and crystal packing has but a small effect on the electronic structure of these molecules.

In contrast, calculations using Mulliken charges strongly depend on the choice of basis set (both the size and the type). With these charges, results with smaller basis sets are closer to the experimental value. They provide reasonable energies with 3-21G(p) and 6-31G(d), but fail to reproduce sensible values if a larger basis set is used $(6-311++G(d, p))$. This is in line with the well-known sensitivity of the Mulliken method to the basis set.

The experimental emission in the solid state has been attributed to the $\mathbf{K}^{*}$ form. Regardless of the higher stability of $\mathbf{K}^{*}$ in the excited state, emission from the $\mathbf{E}^{*}$ form is expected to be close to the initial absorption and consequently reabsorbed. Accordingly, our TDDFT calculations predict emission from $\mathbf{E}^{*}$ in the range of $3.1-3.3 \mathrm{eV}$. Interestingly, emission from the $\mathbf{K}^{*}$ form $(\sim 2.7 \mathrm{eV})$ is significantly deviated from the experimental values $(1.7-1.9 \mathrm{eV}) . \stackrel{43172}{ }$ This is not improved by using self-consistent point charges in the SCPCE- $\mathrm{S}_{1}$ method, which is to be expected due to the localisation of the excited state to one molecule (Figure 5). When the emission is calculated using RI-CC2, the energy is improved but is still deviated by more than $0.5 \mathrm{eV}$ from the experiments.

The most significant factor is the geometry itself, obtained at QM:MM level. We show later that a better emission energy is obtained when optimisation is done using the OEEC and SC-OEEC methods. When it comes to the optimisation of excited state minima and $\mathrm{S}_{1}-\mathrm{S}_{0} \mathrm{MECI}$, the PCE approach was unsuccessful for most charges types, due to the lack of non-Coulombic short-range interactions and ensuing overpolarisation effects. Only Hirshfeld and, in certain cases, crystal RESP charges were overall small enough in magnitude to allow for the determination of local minima. As such optimisation with PCE in general is not recommended for systems with a high degree of conformational flexibility. 
As for $\mathrm{S}_{1}-\mathrm{S}_{0}$ MECI energies, all TDDFT results for the QM:MM geometries are more than $1 \mathrm{eV}$ above the FC bright state energy (Table 2). The conical intersections are thus rendered inaccessible as expected since $\mathbf{H C 1}$ displays aggregation-induced emission. The energies obtained with $\mathrm{RI}-\mathrm{CC} 2$ are in the range of $0.6-0.7 \mathrm{eV}$ above their corresponding excitation energies which also makes them inaccessible. These results are consistent within the RACI model but are overestimated with respect to the value of $3.97 \mathrm{eV}$ obtained with QM:MM with a dimer in the QM region. ${ }^{42}$

In the case of RESP charges, optimisation within the PCE model does not significantly change the energetics previously evaluated with single point calculations. Indeed the structures are close to those reported at the QM:MM level of theory. The resulting relative energies are shown in Figure 6. For Hirshfeld charges, the effect of optimisation is more significant reducing the $\mathrm{K}^{*}$ emission energies to $2.28 \mathrm{eV}$ and making the $\mathrm{S}_{1}-\mathrm{S}_{0}$ MECI accessible.

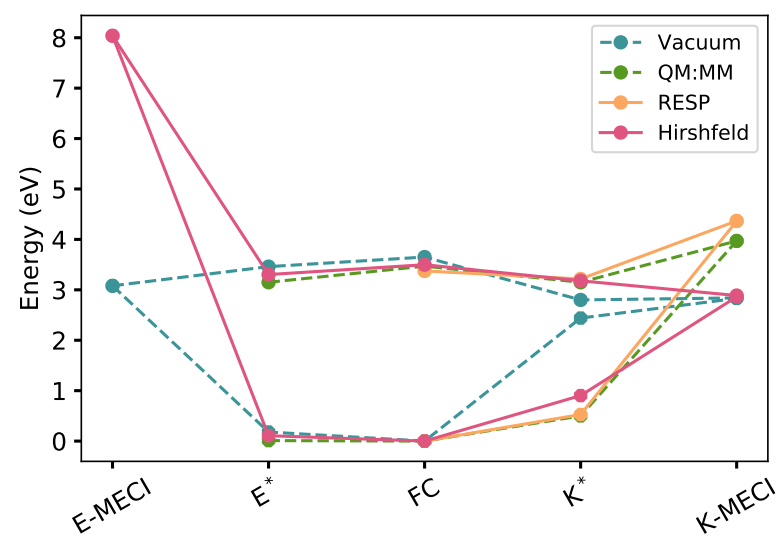

Figure 6: Energies HC1 in vacuum and embedded in RESP and crystal Hirshfeld Ewald charges at different geometries. QM:MM energies were taken from Ref 42 .

Comparing the calculations in $\mathrm{PCE}$ and vacuum at the same geometry highlights the main effects of the crystal electrostatic environment in the excited state. The excited states are overall stabilised which reduces both the vertical excitation and conical intersection energies. However the accessibility of the latter depends on the former, netting no clear difference in emissive behaviour. A more substantial relative stabilisation of the MECI is observable when the molecule is fully optimised in vacuum. It reaches a highly distorted 
geometry which would be inaccessible in the solid due to short-range interactions of the closely packed neighbouring molecules.

Our simulations show some of the drawbacks of the PCE method, in particular for its use in geometry optimisation. Because of the effects of overpolarisation and the lack of shortrange non-Coulombic interactions, electrostatic forces can become too large and some nuclear configurations become unstable. Consequently, we do not recommend the use of PCE for

geometry optimisations. While the method is effective in some cases, ${ }^{75576}$ it is unpredictable whether it will provide reliable geometries for all regions of the PES. To mitigate these problems we implemented a two level embedded cluster model.

\subsection{Embedded Cluster Models: Potential Energy Surfaces in the Crystal}

We obtained the geometries of notable regions of the PES for $\mathbf{H C 1}$ and $\mathbf{H C 2}$ crystals using the OEEC and its self-consistent variant SC-OEEC methods. Table 3 shows the absorption and emission energies obtained after optimisation of $\mathbf{F C}$ and $\mathbf{K}^{*}$ forms using these models.

Table 3: Table of absorption and emission energies for both model systems after geometry optimisation with cluster models. The level of theory was TD- $\omega$ B97X-D/6-311++G(d,p). Energies are in $\mathrm{eV} .{ }^{\dagger}$ Charges obtained for the $\mathbf{K}^{*}$ form in $\mathrm{S}_{1}$

\begin{tabular}{|c|c|c|c|c|}
\hline \multirow{2}{*}{ Cluster model } & \multicolumn{2}{|r|}{ HC1 } & \multicolumn{2}{|c|}{$\mathrm{HC} 2$} \\
\hline & FC & $\mathrm{K}^{*}$ & FC & $\mathbf{K}^{*}$ \\
\hline OEEC & 3.37 & 2.06 & 3.41 & 2.07 \\
\hline SC-OEEC-S ${ }_{1}$ & 3.08 & $2.60(2.12)^{\dagger}$ & 3.34 & 2.15 \\
\hline SC-OEEC-S ${ }_{0}$ & 3.27 & 2.19 & 3.40 & 2.07 \\
\hline OEC & 3.27 & 2.40 & 3.42 & 2.03 \\
\hline $\mathrm{PCM}$ & 3.32 & 2.36 & 3.72 & 2.44 \\
\hline SC-PCM & 3.00 & 2.66 & 3.01 & 2.21 \\
\hline ONIOM QM:MM (molecule) 42 & 3.32 & 2.72 & 3.50 & 2.17 \\
\hline ONIOM QM:MM (dimer) & 3.27 & 2.61 & 3.29 & 2.19 \\
\hline 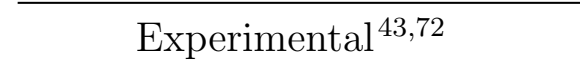 & $2.9,3.3$ & $1.7-1.9$ & - & 1.8 \\
\hline
\end{tabular}


Geometry optimisation using the embedded cluster method produces ground state geometries very similar geometries to QM:MM, consequently the absorption energies are not significantly altered between PCE and OEEC or SC-PCE-S 1 and SC-OEEC-S 1 provided that RESP charges are used throughout (Table 2). For comparison, we have added the results obtained with ONIOM (QM:MM) including one and two molecules in the QM region and with the OEC model. Moreover, we present results in PCM and its self-consistent variant, both in DCM solvent, since continuum models are a common method employed to reflect the electrostatic environment in molecular condensed matter. .77

For all cluster models, the effect of the polarised response of the environment is to reduce the vertical excitation. This is also observed in the comparison between the PCM and SCPCM models and when the size of the QM region increases from a molecule to dimer. The results obtained with the SC-OEEC procedure depend on the level of excitation in the selfconsistent loop. If ground state charges are used, as expected, the energies are similar to those obtained with OEEC (3.27 and $3.37 \mathrm{eV}$ for $\mathbf{H C 1}$ and 3.40 and 3.41 for HC2). The emission energies obtained with different methods strongly depend on the rotation angle (Figure 1). In vacuum, the excited state minima show a significant deviation from their ground state planar structure. In the solid state, the $\mathbf{K}^{*}$ geometries obtained with QM:MM and SC-OEEC-S $\mathrm{S}_{1}$ are more planar $\left(\mathbf{H C 1}: 6^{\circ}\right.$ and $9^{\circ}, \mathbf{H C}$ : $16^{\circ}$ and $12^{\circ}$ respectively for the angle depicted in Figure 1) than those obtained with OEEC (HC1: $32^{\circ}, \mathbf{H C 2}$ : $\left.18^{\circ}\right)$.

The optimisation of the $\mathbf{K}^{*}$ form with the EEC model significantly improves the emission energies with respect to those obtained using QM:MM geometries. Scheme 1 summarises the deviation of the calculated emission energies with respect to the experimental data.

For $\mathbf{H C 1}$, the result is $2.06 \mathrm{eV}$ (TD- $\omega \mathrm{B} 97 \mathrm{X}-\mathrm{D} / 6-311++\mathrm{G}(\mathrm{d}, \mathrm{p}))$, which is in close agreement with the experimental value. As with the PCE method, the self-consistent background based on the excited state charges at the $\mathbf{F C}$ state does not improve the results $(2.6 \mathrm{eV})$. If ground state $\mathbf{E}$ charges are employed in the self-consistent loop, the emission energies remain in better agreement with the experimental values. Indeed, given the level of localisa- 


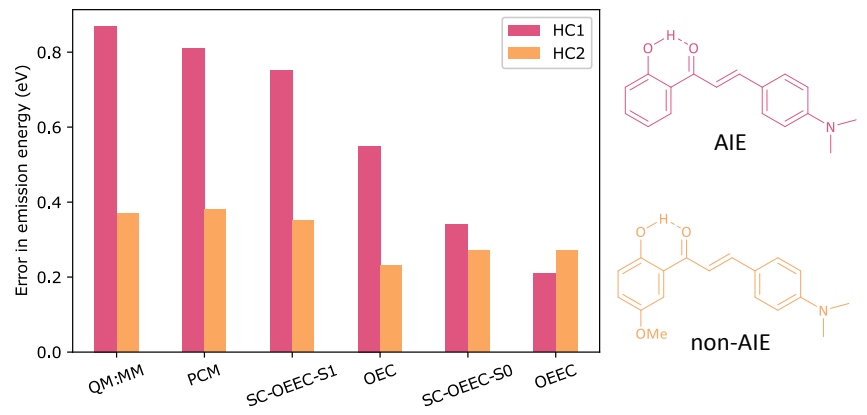

Scheme 1: Deviation of the predicted emission energies of HC1 and HC2 with respect the experimental values by different embedding models. Reference experimental values were 1.8 eV for both HC1 and HC2 crystals

tion of the excitation in these systems (Figure 5), the use of ground state charges seems to be more appropriate $\left(\mathrm{SC}-\mathrm{OEEC}-\mathrm{S}_{0}\right)$ with emission energy of $2.19 \mathrm{eV}$. An alternate version of SC-OEEC-S $\mathrm{S}_{1}$ is also employed where the self-consistent loop is carried out on a molecule in its OEEC optimised $\mathbf{K}^{*}$ geometry, which brings the emission energy to $2.12 \mathrm{eV}$. However, this charge background represents the situation where all molecules in the crystal exhibit charges from the keto form, which is highly unlikely since this would not represent a low energy excited state.

Comparison of the OEEC with the OEC model indicates that long-range interactions account for more than $0.3 \mathrm{eV}$ in the $\mathbf{K}^{*}$ emission energy of $\mathbf{H C 1}$. Interestingly, while the energies for HC1 strongly depend on the charge background, the values for HC2 are less affected. In the case of $\mathbf{H C 2}$, SC-OEEC, OEEC and OEC all provided very similar results $(2.15,2.07$ and $2.07 \mathrm{eV})$, suggesting that the most important Coulomb effects are recovered at the short-range. This is linked to the difference between the ground and excited state charges of these molecules. For HC1, excitation significantly alters the charges of carbon atoms in the bridge (Supporting Information). In the case of HC2 only the charge of the carbonyl carbon $\left(\mathrm{C}_{k}\right)$ changes more than $0.1 e^{-}$upon excitation. Consequently, the $\mathrm{S}_{1}-\mathrm{S}_{0}$ energy gaps for HC1 are far more dependent on the electrostatic environment. Indeed improving the description of the short-range intermolecular interactions does not significantly alter the energy gaps, as illustrated by the behaviour of the absorption and emission energies with 
the size of the QM region (see end of section). For both, HC1 and HC2, the emission from $\mathbf{K}^{*}$ is fairly well reproduced with only one molecule in the QM region, which is in line with the localised nature of the $\mathbf{K}^{*}$ (Figure 5).

Conical intersections play a key role in photophenomena, providing a radiationless decay funnel for the excited state. One of the features implemented in fromage is the searching of crossing geometries using the penalty function method of Levine et al.. $\stackrel{52}{\text { The molecules }}$ considered here can deactivate to the ground state in solution via conical intersections associated with intramolecular rotation. $\frac{4142}{4 e}$ optimise the $\mathrm{S}_{1}-\mathrm{S}_{0}$ MECI with the SA-2CASSCF $(12,11) / 6-31 G(d)$ and TD- $\omega$ B97X-D/6-311++G(d,p) methods within the OEEC scheme (Figure 7). For these systems, the geometries obtained with both levels of theory are in very good agreement.

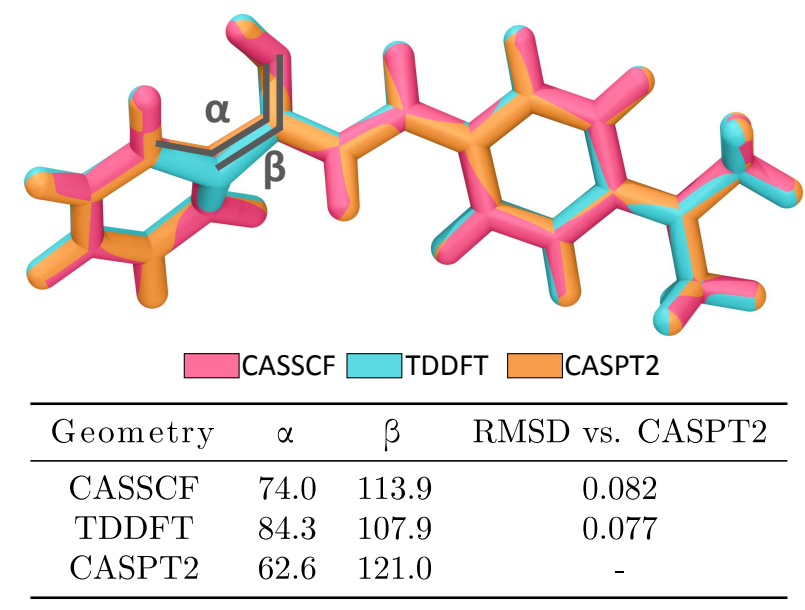

Figure 7: MECI geometries found with SA-2-CASSCF $(12,11) / 6-31 G(d)$ and TD- $\omega$ B97X$\mathrm{D} / 6-311++\mathrm{G}(\mathrm{d}, \mathrm{p})$. Additionally, we include the configuration with least $\mathrm{S}_{1}-\mathrm{S}_{0}$ gap when scanning the $\beta$ angle from the TDDFT geometry at MS-2-CASPT2(12,11)/6-31G(d) level. It is labelled CASPT2.

In the crystal, the lowest energy conical intersection combines intramolecular rotation and a significant pyramidalisation of the carbonyl carbon. ${ }^{42}$ In the gas phase, the lowest energy conical intersection only involves intramolecular rotation while the one also involving pyramidalisation is higher in energy. Therefore one of the effects of the crystal environment is to modify the stability of the lowest energy conical intersections, which is consistent with 
the results obtained with QM:MM calculations. This confirms that the effect of shortrange interaction is essential in determining the geometry while the long-range interactions modulate the total energy. However, the net effect of the embedding on the total energies is highly system dependent.
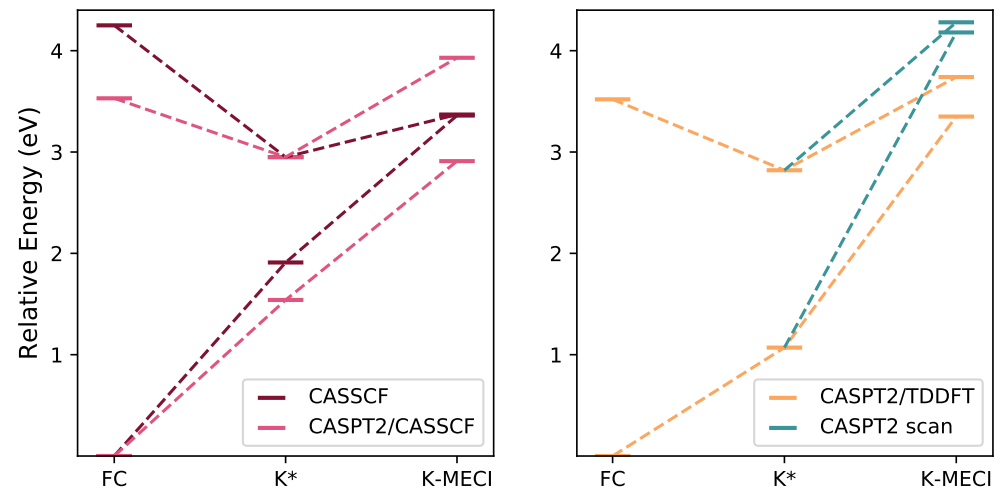

Figure 8: Relative energy diagrams showing the emission energy and accessibility of the MECI with multireference methods at the geometries shown in Figure 7 . To minimise the CASPT2 $\mathrm{S}_{1}-\mathrm{S}_{0}$ gap at MECI configurations, a geometry scan was carried out as described in Figure 9. The newly optimised geometry is labelled "CASPT2 scan" and has a gap of $0.10 \mathrm{eV}$.

Figure 8 shows the PES obtained with multireference methods. The vertical excitation obtained with SA-2-CASSCF $(12,11) / 6-31 \mathrm{G}(\mathrm{d})$ is significantly deviated from the experimental value (4.25 eV compared to $3.3 \mathrm{eV}$ ). Including dynamic electron correlation with MS-2CASPT2 $(12,11) / 6-31 G(d)$ shifted the value to the red in much better agreement with the experimental value $(3.53 \mathrm{eV})$. The energy gap at the CASSCF conical intersection is too large with PT2 $(1.02 \mathrm{eV})$, but using TDDFT geometries as reference can significantly narrow the gap. These are common challenges found in multireference calculations and not due to the embedding approach. $\frac{780}{80}$ In order to further narrow the $\mathrm{S}_{1}-\mathrm{S}_{0}$ gap, the aromatic $\mathrm{C}$ was systematically displaced via the $\beta$ angle (Figure 7). Figure 9 shows how this scan locates a conical intersection at an energy $0.8 \mathrm{eV}$ above the $\mathbf{F C}$ energy. These examples show that the Ewald embedding methods can provide all the information required to fully characterise the PES in molecular crystals. All of these methods are available in fromage. 


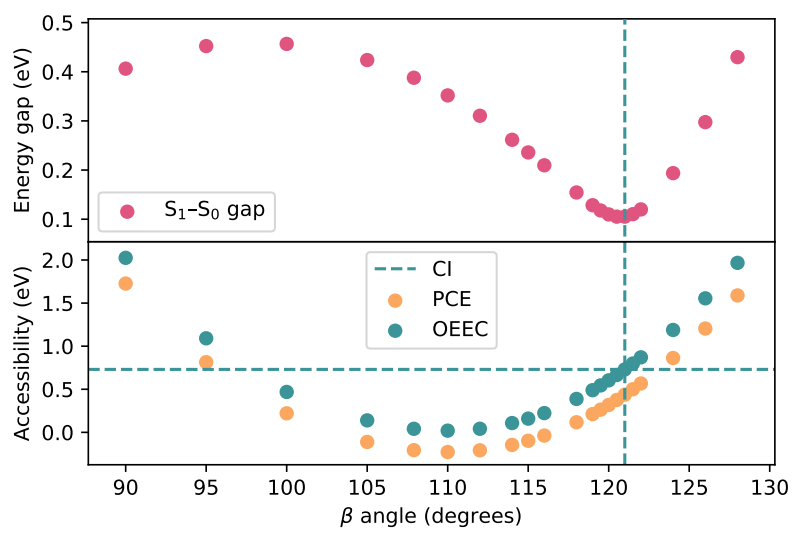

Figure 9: Plot of the $S_{1}-S_{0}$ gap, and the MECI accessibility as a function of the puckering angle $\beta$. The accessibility is defined as the average $\mathrm{S}_{1}-\mathrm{S}_{0}$ energy of the given geometry minus the $\mathbf{F C}$ bright state energy. The OEEC energy at the geometry with smallest $\mathrm{S}_{1}-\mathrm{S}_{0}$ gap is indicated by dashed lines.

Given that the Ewald embedding methods describe the effect of the electrostatics of the whole crystal, they represent unique schemes to analyse the convergence of properties with the size of the QM region. Exploring these effects is essential in systems with significant excitonic effects. We consider the behaviour of the energies and the accessibility of the $\mathrm{S}_{1}-\mathrm{S}_{0}$ MECI with the size of the QM region (Figure 10). We employ the TD- $\omega$ B97X-D/6-31G(d) level of theory, which provides a good description of different regions of the PES. For HC1, the energies of the bright state for $\mathbf{F C}$ and of the emission from $\mathbf{K}^{*}$ converge relatively quickly. On the other hand, the energy of the $\mathrm{S}_{1}-\mathrm{S}_{0}$ MECI increases with the size of the QM region for $\mathbf{H C 1}$ and decreases for $\mathbf{H C 2}$ respectively becoming less and more accessible. This is in line with the experimental behaviour of both crystals.

\section{Conclusion}

In this paper, we analyse the behaviour of different Ewald embedding schemes for the description of excited states in molecular crystals. With focus on the exploration of potential energy surfaces, we have implemented these methods in the Python open-source platform fromage, which we make readily available. This program enables users to easily combine 


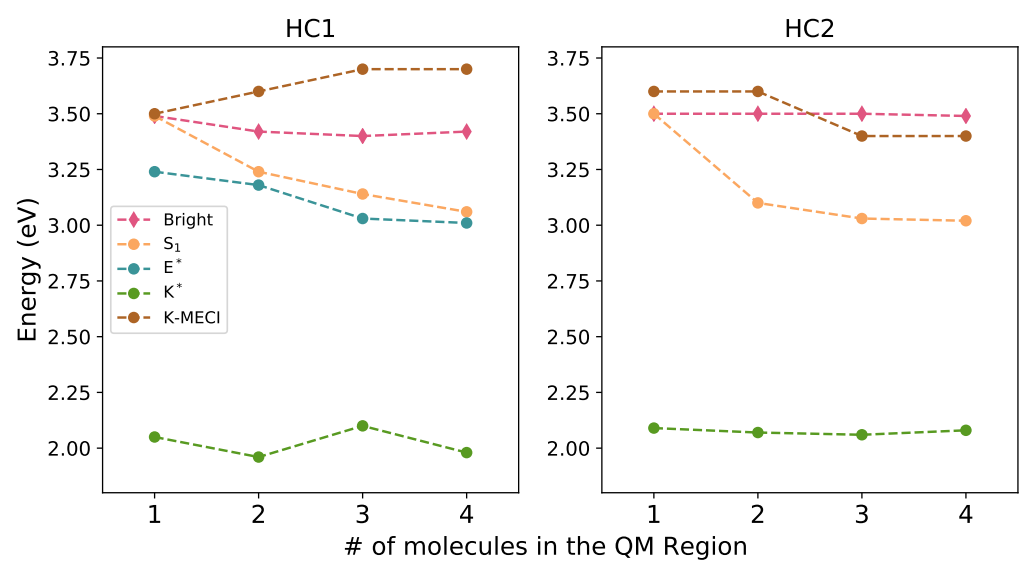

Figure 10: Energy of the absorption, emission and conical intersection as the excited state region increases in size using the OEEC model. The region molecules are added to the region in the order displayed in Figure 4. The energies are evaluated with TD- $\omega$ B97X-D/6-31G(d).

electronic structure codes of their choice for geometry optimisation using OEC, OEEC and SC-OEEC. The current implementation includes interfaces to popular quantum chemistry programs such as Turbomole, Gaussian and Molcas. Additional interfaces can be easily implemented, provided that the new codes allow for gradient calculations with point charge embedding.

We have shown that the PCE method is poorly suited to optimising the geometry of flexible molecules in the crystal form. Consequently, the photochemical conclusions that arise from PCE calculations of such molecules have the potential to be quantitatively and qualitatively erroneous. To overcome this problem, a series of two-level ONIOM(QM:QM') cluster models with Ewald embedding were formulated. They are suitable for geometry optimisation of excited state minima and conical intersections.

The potential of these tools was illustrated by applying them to the excited states of two model crystals, HC1 and HC2, displaying excited state intramolecular proton transfer. HC1 displays aggregation induced emission whilst HC2 shows no emission in solution or solid state. For both systems, the excitations are clearly localised in one or two molecules which allowed the emission energies to converge with only a monomer or a dimer in the QM region. We found that using charges originating from molecular or crystal calculations 
did not significantly impact the results. The emission energy was progressively improved with a hierarchy of embedding models, ranging from a deviation from the experimental emission peak of $0.8 \mathrm{eV}$ with ONIOM QM:MM to $0.2 \mathrm{eV}$ with OEEC. Due to the flexibility of the molecules, this increase in accuracy could only be achieved by carrying out geometry optimisation with each embedding model.

Self-consistent procedures help to model the mutual polarisation between the excited state region and the environment. In particular, if the self-consistent loop is carried out in the excited state, these procedures may help reflect the delocalisation of an excitation despite explicitly modelling fewer excited fragments than are involved in the delocalisation. For the systems considered in this study, the degree of localisation in both the absorption and emission processes made excited state self-consistent embedding unsuitable. The application of the self-consistent procedure to the ground state did not significantly alter the results obtained from the corresponding non-self-consistent procedure which suggests that the electronic structure of the isolated ground state molecule is not particularly altered by crystal packing.

With these conclusions in mind, we can suggest optimal embedding methods for the study of the photochemistry of different molecular crystals. If the molecule is certain to be structurally rigid and the exciton couplings are small, PCE scheme can be appropriate. Otherwise, cluster models are preferred since they allow for exploration of the nuclear configuration space. In either case, long range electrostatic interactions can account for a significant contribution to the excited state energy. Comparison between excitation energies obtain with truncated cluster models and single point calculations with Ewald embedding methods can help decide whether these methods are required.

The size of the QM region should be motivated by the locality of the excitation at the noteworthy points of the PES which can be estimated by the calculation of exciton couplings between molecular fragments in their lattice positions. These coupling values can, in turn, be estimated as half of the $\mathrm{S}_{2}-\mathrm{S}_{1}$ energy gap for the dimer ${ }^{81}$ or by using more sophisticated 
methods (see Supporting Information). For localised excitations, ground state background

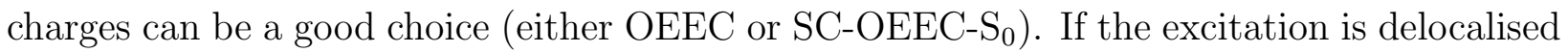
and the QM region becomes impractically large, the SC-OEEC-S ${ }_{1}$ method can provide a better description of the excited states. We believe the use of these methods will contribute to a better understanding of complex photochemical processes in the crystal environment, impacting a broad range of applications.

\section{Acknowledgement}

This work was supported by the EPSRC (EP/R029385/1). This research utilised Queen Mary's Apocrita HPC facility, supported by QMUL Research-IT, ARCHER UK National Supercomputing Service (EP/L000202) via the Materials Chemistry Consortium and the Thomas cluster via our membership to the Thomas Young Centre. We acknowledge the support from the School of Biological and Chemical Sciences at Queen Mary University of London. The authors thank M. Klintenberg and S. E. Derenzo for allowing the modification and redistribution of the Ewald program and A. A. Sokol for meaningful discussion.

\section{Supporting Information Available}

The fromage code can be found at https://github.com/Crespo-Otero-group/fromage and can be contributed to upon request. The modified version of Ewald can be found at https://github.com/Crespo-Otero-group/Ewald.

\section{References}

(1) Li, W.; Pan, Y.; Yao, L.; Liu, H.; Zhang, S.; Wang, C.; Shen, F.; Lu, P.; Yang, B.; Ma, Y. A Hybridized Local and Charge-Transfer Excited State for Highly Efficient Fluorescent 
OLEDs: Molecular Design, Spectral Character, and Full Exciton Utilization. Adv. Opt. Mater. 2014, 2, 892-901.

(2) Jo, S. B.; Kim, H. H.; Lee, H.; Kang, B.; Lee, S.; Sim, M.; Kim, M.; Lee, W. H.; Cho, K. Boosting Photon Harvesting in Organic Solar Cells With Highly Oriented Molecular Crystals via Graphene-Organic Heterointerface. ACS Nano 2015, 9, 8206-8219.

(3) Gierschner, J.; Varghese, S.; Park, S. Y. Organic Single Crystal Lasers: A Materials View. Adv. Opt. Mater. 2016, 4, 348-364.

(4) Aragó, J.; Troisi, A. Dynamics of the Excitonic Coupling in Organic Crystals. Phys. Rev. Lett. 2015, 114, 026402.

(5) Severo Pereira Gomes, A.; Jacob, C. R. Quantum-Chemical Embedding Methods for Treating Local Electronic Excitations in Complex Chemical Systems. Annu. Rep. Prog. Chem. 2012, 108, 222-277.

(6) Kochman, M. A.; Bil, A.; Morrison, C. A. Hybrid QM/QM Simulations of Photochemical Reactions in the Molecular Crystal N-Salicylidene-2-Chloroaniline. Phys. Chem. Chem. Phys. 2013, 15, 10803-10806.

(7) Kochman, M. A.; Morrison, C. A. Hybrid QM/QM Simulations of Excited-State Intramolecular Proton Transfer in the Molecular Crystal 7-(2-Pyridyl)-Indole. J. Chem. Theory Comput. 2013, 9, 1182-1192.

(8) Libisch, F.; Huang, C.; Carter, E. A. Embedded Correlated Wavefunction Schemes: Theory and Applications. Acc. Chem. Res. 2014, 47, 2768-2775.

(9) Cheng, J.; Yu, K.; Libisch, F.; Dieterich, J. M.; Carter, E. A. Potential Functional Embedding Theory at the Correlated Wave Function Level. 2. Error Sources and Performance Tests. J. Chem. Theory Comput. 2017, 13, 1081-1093. 
(10) Cheng, J.; Libisch, F.; Yu, K.; Chen, M.; Dieterich, J. M.; Carter, E. A. Potential Functional Embedding Theory at the Correlated Wave Function Level. 1. Mixed Basis Set Embedding. J. Chem. Theory Comput. 2017, 13, 1067-1080.

(11) Presti, D.; Labat, F.; Pedone, A.; Frisch, M. J.; Hratchian, H. P.; Ciofini, I.; Menziani, M. C.; Adamo, C. Computational Protocol for Modeling Thermochromic Molecular Crystals: Salicylidene Aniline as a Case Study. J. Chem. Theory Comput. 2014, 10, 5577-5585.

(12) Presti, D.; Pedone, A.; Ciofini, I.; Labat, F.; Menziani, M. C.; Adamo, C. Optical Properties of the Dibenzothiazolylphenol Molecular Crystals Through ONIOM Calculations: The Effect of the Electrostatic Embedding Scheme. Theor. Chem. Acc. 2016, 135,86 .

(13) Presti, D.; Labat, F.; Pedone, A.; Frisch, M. J.; Hratchian, H. P.; Ciofini, I.; Cristina Menziani, M.; Adamo, C. Modeling Emission Features of Salicylidene Aniline Molecular Crystals: A QM/QM' Approach. J. Comput. Chem. 2016, 37, 861-870.

(14) Kantorovich, L. Quantum Theory of the Solid State: An Introduction; Springer Netherlands: Dordrecht, 2004.

(15) Weber, J.; Schmedt auf der Günne, J. Calculation of NMR Parameters in Ionic Solids by an Improved Self-Consistent Embedded Cluster Method. Phys. Chem. Chem. Phys. 2010, 12, 583-603.

(16) Torras, J.; Bromley, S.; Bertran, O.; Illas, F. Modelling Organic Molecular Crystals by Hybrid Quantum Mechanical/Molecular Mechanical Embedding. Chem. Phys. Lett. 2008, 457, 154-158.

(17) McKenna, K. P.; Sushko, P. V.; Shluger, A. L. Inside Powders: A Theoretical Model of Interfaces Between MgO Nanocrystallites. J. Am. Chem. Soc. 2007, 129, 8600-8608. 
(18) Kittel, C. Introduction to Solid State Physics; John Wiley \& Sons, Inc.: New York, 2005; Vol. 8th ed.

(19) Toukmaji, A. Y.; Board, J. a. Ewald Summation Techniques in Perspective: A Survey. Comput. Phys. Commun. 1996, 95, 73-92.

(20) Sauer, J. Molecular Models in Ab Initio Studies of Solids and Surfaces: From Ionic Crystals and Semiconductors to Catalysts. Chem. Rev. 1989, 89, 199-255.

(21) Klintenberg, M.; Derenzo, S.; Weber, M. Accurate Crystal Fields for Embedded Cluster Calculations. Comput. Phys. Commun. 2000, 131, 120-128.

(22) Derenzo, S. E.; Klintenberg, M. K.; Weber, M. J. Determining Point Charge Arrays That Produce Accurate Ionic Crystal Fields for Atomic Cluster Calculations. J. Chem. Phys. 2000, 112, 2074-2081.

(23) Jug, K.; Bredow, T. Models for the Treatment of Crystalline Solids and Surfaces. J. Comput. Chem. 2004, 25, 1551-1567.

(24) Stueber, D.; Guenneau, F. N.; Grant, D. M. The Calculation of 13C Chemical Shielding Tensors in Ionic Compounds Utilizing Point Charge Arrays Obtained From Ewald Lattice Sums. J. Chem. Phys. 2001, 114, 9236-9243.

(25) Sokol, A. A.; Bromley, S. T.; French, S. A.; Catlow, C. R. A.; Sherwood, P. Hybrid QM/MM Embedding Approach for the Treatment of Localized Surface States in Ionic Materials. Int. J. Quantum Chem. 2004, 99, 695-712.

(26) Metz, S.; Kästner, J.; Sokol, A. A.; Keal, T. W.; Sherwood, P. ChemShell-a Modular Software Package for QM/MM Simulations. Wiley Interdiscip. Rev. Comput. Mol. Sci. 2014, 4, 101-110.

(27) Abarenkov, I. V. Unit Cell for a Lattice Electrostatic Potential. Phys. Rev. B 2007, $76,165127$. 
(28) Sushko, P. V.; Abarenkov, I. V. General Purpose Electrostatic Embedding Potential. J. Chem. Theory Comput. 2010, 6, 1323-1333.

(29) Kobayashi, O.; Nanbu, S. Application of Particle-Mesh Ewald Summation to ONIOM Theory. Chem. Phys. 2015, 461, 47-57.

(30) Nam, K.; Gao, J.; York, D. M. An Efficient Linear-Scaling Ewald Method for LongRange Electrostatic Interactions in Combined QM/MM Calculations. J. Chem. Theory Comput. 2005, 1, 2-13.

(31) Wang, Y.; Lopata, K.; Chambers, S. A.; Govind, N.; Sushko, P. V. Optical Absorption and Band Gap Reduction in (Fe1-xCrx)2O3 Solid Solutions: A First-Principles Study. J. Phys. Chem. C 2013, 117, 25504-25512.

(32) Sushko, P. V.; Qiao, L.; Bowden, M.; Varga, T.; Exarhos, G. J.; Urban, F. K.; Barton, D.; Chambers, S. A. Multiband Optical Absorption Controlled by Lattice Strain in Thin-Film LaCrO3. Phys. Rev. Lett. 2013, 110, 077401.

(33) Sousa, C.; Tosoni, S.; Illas, F. Theoretical Approaches to Excited-State-Related Phenomena in Oxide Surfaces. Chem. Rev. 2013, 113, 4456-4495.

(34) Cui, G.; Yang, W. Conical Intersections in Solution: Formulation, Algorithm, and Implementation With Combined Quantum Mechanics/Molecular Mechanics Method. J. Chem. Phys. 2011, 134, 204115.

(35) Wilbraham, L.; Adamo, C.; Labat, F.; Ciofini, I. Electrostatic Embedding to Model the Impact of Environment on Photophysical Properties of Molecular Crystals: A SelfConsistent Charge Adjustment Procedure. J. Chem. Theory Comput. 2016, 12, 33163324 .

(36) Presti, D.; Wilbraham, L.; Targa, C.; Labat, F.; Pedone, A.; Menziani, M. C.; Ciofini, I.; 
Adamo, C. Understanding Aggregation-Induced Emission in Molecular Crystals: Insights From Theory. J. Phys. Chem. C 2017, 121, 5747-5752.

(37) Wilbraham, L.; Louis, M.; Alberga, D.; Brosseau, A.; Guillot, R.; Ito, F.; Labat, F.; Métivier, R.; Allain, C.; Ciofini, I. Revealing the Origins of Mechanically Induced Fluorescence Changes in Organic Molecular Crystals. Adv. Mater. 2018, 30, 1800817.

(38) Cieplak, P.; Dupradeau, F.-Y.; Duan, Y.; Wang, J. Polarization Effects in Molecular Mechanical Force Fields. J. Phys. Condens. Matter 2009, 21, 333102.

(39) Warshel, A.; Kato, M.; Pisliakov, A. V. Polarizable Force Fields: History, Test Cases, and Prospects. J. Chem. Theory Comput. 2007, 3, 2034-2045.

(40) Xie, W.; Gao, J. Design of a Next Generation Force Field: The X-Pol Potential. J. Chem. Theory Comput. 2007, 3, 1890-1900.

(41) Dommett, M.; Crespo-Otero, R. Excited State Proton Transfer in 2'-Hydroxychalcone Derivatives. Phys. Chem. Chem. Phys. 2017, 19, 2409-2416.

(42) Dommett, M.; Rivera, M.; Crespo-Otero, R. How Inter- And Intramolecular Processes Dictate Aggregation-Induced Emission in Crystals Undergoing Excited-State Proton Transfer. J. Phys. Chem. Lett. 2017, 8, 6148-6153.

(43) Cheng, X.; Wang, K.; Huang, S.; Zhang, H.; Zhang, H.; Wang, Y. Organic Crystals With Near-Infrared Amplified Spontaneous Emissions Based on 2'-Hydroxychalcone Derivatives: Subtle Structure Modification but Great Property Change. Angew. Chem. Int. Ed. 2015, 54, 8369-8373.

(44) Crespo-Otero, R.; Li, Q.; Blancafort, L. Exploring Potential Energy Surfaces for Aggregation-Induced Emission - from Solution to Crystal. Chem. Asian J. 2018, asia.201801649. 
(45) Li, Q.; Blancafort, L. A Conical Intersection Model to Explain Aggregation Induced Emission in Diphenyl Dibenzofulvene. ChemComm 2013, 49, 5966-5968.

(46) Rivera, M.; Dommett, M.; Crespo-Otero, R. fromage: A FRamewOrk for Molecular AGgregate Excitations. 2018; https://github.com/Crespo-Otero-group/fromage.

(47) Rivera, M.; Dommett, M.; Crespo-Otero, R. Documentation for fromage. 2018; https: //fromage.readthedocs.io/.

(48) Frisch, M. J.; Trucks, G. W.; Schlegel, H. B.; Scuseria, G. E.; Robb, M. A.; Cheeseman, J. R.; Scalmani, G.; Barone, V.; Mennucci, B.; Petersson, G. A.; Nakatsuji, H.; Caricato, M.; Li, X.; Hratchian, H. P.; Izmaylov, A. F.; Bloino, J.; Zheng, G.; Sonnenberg, J. L.; Hada, M.; Ehara, M.; Toyota, K.; Fukuda, R.; Hasegawa, J.; Ishida, M.; Nakajima, T.; Honda, Y.; Kitao, O.; Nakai, H.; Vreven, T.; Montgomery, J. A., Jr.; Peralta, J. E.; Ogliaro, F.; Bearpark, M.; Heyd, J. J.; Brothers, E.; Kudin, K. N.; Staroverov, V. N.; Kobayashi, R.; Normand, J.; Raghavachari, K.; Rendell, A.; Burant, J. C.; Iyengar, S. S.; Tomasi, J.; Cossi, M.; Rega, N.; Millam, J. M.; Klene, M.; Knox, J. E.; Cross, J. B.; Bakken, V.; Adamo, C.; Jaramillo, J.; Gomperts, R.; Stratmann, R. E.; Yazyev, O.; Austin, A. J.; Cammi, R.; Pomelli, C.; Ochterski, J. W.; Martin, R. L.; Morokuma, K.; Zakrzewski, V. G.; Voth, G. A.; Salvador, P.; Dannenberg, J. J.; Dapprich, S.; Daniels, A. D.; Farkas, O.; Foresman, J. B.; Ortiz, J. V.; Cioslowski, J.; Fox, D. J. Gaussian09 Revision E.01. Gaussian Inc. Wallingford CT 2009.

(49) Aquilante, F.; Autschbach, J.; Carlson, R. K.; Chibotaru, L. F.; Delcey, M. G.; De Vico, L.; Fdez Galván, I.; Ferré, N.; Frutos, L. M.; Gagliardi, L.; Garavelli, M.; Giussani, A.; Hoyer, C. E.; Li Manni, G.; Lischka, H.; Ma, D.; Malmqvist, P. Å.; Müller, T.; Nenov, A.; Olivucci, M.; Pedersen, T. B.; Peng, D.; Plasser, F.; Pritchard, B.; Reiher, M.; Rivalta, I.; Schapiro, I.; Segarra-Martí, J.; Stenrup, M.; Truhlar, D. G.; Ungur, L.; Valentini, A.; Vancoillie, S.; Veryazov, V.; Vysotskiy, V. P.; Weingart, O.; 
Zapata, F.; Lindh, R. Molcas 8: New Capabilities for Multiconfigurational Quantum Chemical Calculations Across the Periodic Table. J. Comput. Chem. 2016, 37, 506-541.

(50) TURBOMOLE V6.2 2010, a development of University of Karlsruhe and Forschungszentrum Karlsruhe GmbH, 1989-2007, TURBOMOLE GmbH, since 2007; available from http://www.turbomole.com.

(51) Aradi, B.; Hourahine, B.; Frauenheim, T. DFTB+, a Sparse Matrix-Based Implementation of the DFTB Method. J. Phys. Chem. B A 2007, 111, 5678-5684.

(52) Levine, B. G.; Coe, J. D.; Martínez, T. J. Optimizing Conical Intersections Without Derivative Coupling Vectors: Application to Multistate Multireference Second-Order Perturbation Theory (MS-CASPT2). J. Phys. Chem. B 2008, 112, 405-413.

(53) Ruiz-Barragan, S.; Morokuma, K.; Blancafort, L. Conical Intersection Optimization Using Composed Steps Inside the ONIOM(QM:MM) Scheme: CASSCF:UFF Implementation with Microiterations. J. Chem. Theory Comput. 2015, 11, 1585-1594, PMID: 26574368.

(54) Levine, B. G.; Martínez, T. J. Isomerization Through Conical Intersections. Annu. Rev. Phys. Chem. 2007, 58, 613-634.

(55) Barbatti, M.; Ruckenbauer, M.; Plasser, F.; Pittner, J.; Granucci, G.; Persico, M.; Lischka, H. Newton-X: A Surface-Hopping Program for Nonadiabatic Molecular Dynamics. Wiley Interdiscip. Rev. Comput. Mol. Sci. 2014, 4, 26-33.

(56) Gozem, S.; Melaccio, F.; Valentini, A.; Filatov, M.; Huix-Rotllant, M.; Ferré, N.; Frutos, L. M.; Angeli, C.; Krylov, A. I.; Granovsky, A. A.; Lindh, R.; Olivucci, M. Shape of Multireference, Equation-Of-Motion Coupled-Cluster, and Density Functional Theory Potential Energy Surfaces at a Conical Intersection. J. Chem. Theory Comput. 2014, 10, 3074-3084. 
(57) Tuna, D.; Lefrancois, D.; Wolański, Ł.; Gozem, S.; Schapiro, I.; Andruniów, T.; Dreuw, A.; Olivucci, M. Assessment of Approximate Coupled-Cluster and AlgebraicDiagrammatic-Construction Methods for Ground- and Excited-State Reaction Paths and the Conical-Intersection Seam of a Retinal-Chromophore Model. J. Chem. Theory Comput. 2015, 11, 5758-5781.

(58) Crespo-Otero, R.; Mardykov, A.; Sanchez-Garcia, E.; Sander, W.; Barbatti, M. Photo-Stability of Peptide-Bond Aggregates: N-Methylformamide Dimers. Phys. Chem. Chem. Phys. 2014, 16, 18877-18887.

(59) Barbatti, M.; Crespo-Otero, R. Surface Hopping Dynamics With DFT Excited States. Top. Curr. Chem. 2016, 368, 415-444.

(60) Chung, L. W.; Sameera, W. M. C.; Ramozzi, R.; Page, A. J.; Hatanaka, M.; Petrova, G. P.; Harris, T. V.; Li, X.; Ke, Z.; Liu, F.; Li, H. B.; Ding, L.; Morokuma, K. The ONIOM Method and Its Applications. Chem. Rev. 2015, 115, 5678-5796.

(61) Presti, D.; Labat, F.; Pedone, A.; Frisch, M. J.; Hratchian, H. P.; Ciofini, I.; Cristina Menziani, M.; Adamo, C. Modeling Emission Features of Salicylidene Aniline Molecular Crystals: A QM/QM'Approach. J. Comput. Chem. 2016, 37, 861-870.

(62) Clemente, F. R.; Vreven, T.; Frisch, M. J. Quantum Biochemistry; John Wiley \& Sons, Ltd, 2010; Chapter 2, pp 61-83.

(63) Biancardi, A.; Barnes, J.; Caricato, M. Point charge embedding for ONIOM excited states calculations. J. Chem. Phys. 2016, 145, 224109.

(64) Kepenekian, M.; Robert, V.; Le Guennic, B.; De Graaf, C. Energetics of $[\mathrm{Fe}(\mathrm{NCH}) 6] 2+$ via CASPT2 Calculations: A Spin-Crossover Perspective. J. Comput. Chem. 2009, 30, 2327-2333. 
(65) Le Guennic, B.; Borshch, S.; Robert, V. Prussian Blue Analogue CsFe[Cr(CN)6] as a Matrix for the Fe(II) Spin-Crossover. Inorg. Chem. 2007, 46, 11106-11111.

(66) Giannozzi, P.; Baroni, S.; Bonini, N.; Calandra, M.; Car, R.; Cavazzoni, C.; Ceresoli, D.; Chiarotti, G. L.; Cococcioni, M.; Dabo, I.; Dal Corso, A.; de Gironcoli, S.; Fabris, S.; Fratesi, G.; Gebauer, R.; Gerstmann, U.; Gougoussis, C.; Kokalj, A.; Lazzeri, M.; Martin-Samos, L.; Marzari, N.; Mauri, F.; Mazzarello, R.; Paolini, S.; Pasquarello, A.; Paulatto, L.; Sbraccia, C.; Scandolo, S.; Sclauzero, G.; Seitsonen, A. P.; Smogunov, A.; Umari, P.; Wentzcovitch, R. M. QUANTUM ESPRESSO: A Modular and Open-Source Software Project for Quantum Simulations of Materials. J. Phys. Condens. Matter 2009, 21, 395502.

(67) Hutter, J.; Iannuzzi, M.; Schiffmann, F.; VandeVondele, J. CP2K: Atomistic Simulations of Condensed Matter Systems. Wiley Interdiscip. Rev. Comput. Mol. Sci. 2014, $4,15-25$.

(68) Henkelman, G.; Arnaldsson, A.; Jónsson, H. A Fast and Robust Algorithm for Bader Decomposition of Charge Density. Comput. Mater. Sci. 2006, 36, 354-360.

(69) Sanville, E.; Kenny, S. D.; Smith, R.; Henkelman, G. Improved Grid-Based Algorithm for Bader Charge Allocation. J. Comput. Chem. 2007, 28, 899-908.

(70) Yu, M.; Trinkle, D. R. Accurate and Efficient Algorithm for Bader Charge Integration. J. Chem. Phys. 2011, 134, 064111.

(71) Tang, W.; Sanville, E.; Henkelman, G. A Grid-Based Bader Analysis Algorithm Without Lattice Bias. J. Phys. Condens. Matter 2009, 21, 084204.

(72) Zahid, N. I.; Mahmood, M. S.; Subramanian, B.; Mohd Said, S.; Abou-Zied, O. K. New Insight Into the Origin of the Red/Near-Infrared Intense Fluorescence of a Crystalline 2-Hydroxychalcone Derivative: A Comprehensive Picture From the Excited-State Femtosecond Dynamics. J. Phys. Chem. Lett. 2017, 8, 5603-5608. 
(73) Crespo-Otero, R.; Barbatti, M. Spectrum Simulation and Decomposition With Nuclear Ensemble: Formal Derivation and Application to Benzene, Furan and 2-Phenylfuran. Theor. Chem. Acc. 2012, 131, 1-14.

(74) Barbatti, M.; Granucci, G.; Ruckenbauer, M.; Plasser, F.; Crespo-Otero, R.; Pittner, J.; Persico, M.; Lischka, NEWTON-X: A Package for Newtonian Dynamics Close to the Crossing Seam. Version 2. 2016; www.newtonx.org.

(75) Wilbraham, L.; Savarese, M.; Rega, N.; Adamo, C.; Ciofini, I. Describing Excited State Intramolecular Proton Transfer in Dual Emissive Systems: A Density Functional Theory Based Analysis. J. Phys. Chem. B 2015, 119, 2459-2466.

(76) Presti, D.; Wilbraham, L.; Targa, C.; Labat, F.; Pedone, A.; Menziani, M. C.; Ciofini, I.; Adamo, C. Understanding Aggregation-Induced Emission in Molecular Crystals: Insights From Theory. J. Phys. Chem. B 2017, 121, 5747-5752.

(77) Skelton, J. M.; Lora da Silva, E.; Crespo-Otero, R.; Hatcher, L. E.; Raithby, P. R.; Parker, S. C.; Walsh, A. Electronic Excitations in Molecular Solids: Bridging Theory and Experiment. Faraday Discuss. 2015, 177, 181-202.

(78) Peng, X.-L.; Ruiz-Barragan, S.; Li, Z.-S.; Li, Q.-S.; Blancafort, L. Restricted Access to a Conical Intersection to Explain Aggregation Induced Emission in Dimethyl Tetraphenylsilole. J. Mat. Chem. C 2016, 4, 2802-2810.

(79) Crespo-Otero, R.; Barbatti, M. Recent Advances and Perspectives on Nonadiabatic Mixed Quantum-Classical Dynamics. Chem. Rev. 2018, 118, 7026-7068.

(80) Barbatti, M.; Lan, Z.; Crespo-Otero, R.; Szymczak, J. J.; Lischka, H.; Thiel, W. Critical Appraisal of Excited State Nonadiabatic Dynamics Simulations of 9H-Adenine. J. Chem. Phys. 2012, 137, 22A503. 
(81) Spano, F. C.; Beljonne, D. In The WSPC Reference on Organic Electronics: Organic Semiconductors; Bredas, J.-L., Marder, S. R., Eds.; World Scientific: Hackensack, 2016; Chapter 4, pp 93-130. 


\section{Graphical TOC Entry}

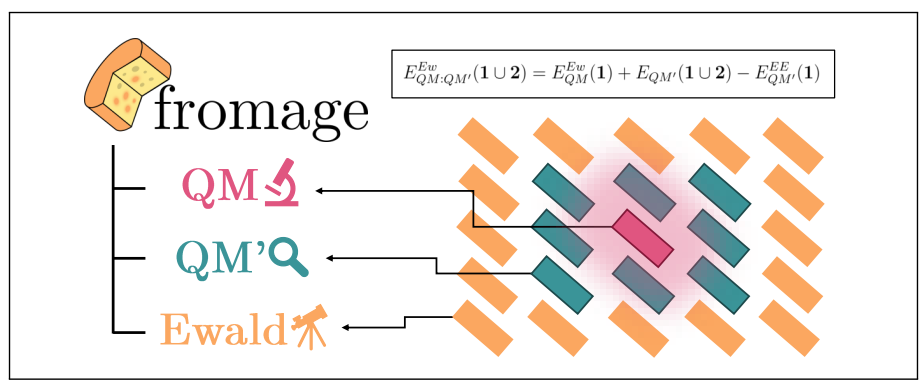

